\title{
NEUROMORPHIC SYSTEM WITH NOVEL ELECTRONIC SYNAPSES BASED ON RRAM DEVICES
}

\author{
${ }^{1}$ ZHIPING ZHANG, ${ }^{2}$ BIN GAO, ${ }^{3}$ XINPENG WANG, ${ }^{4}$ PATRICK LO \\ 1,2,3,4 Institute of Microelectronics, A-STAR, Singapore,Peking University, Beijing, China \\ E-mail: zhangzp@ime.a-star.edu.sg
}

\begin{abstract}
This paper proposes a neuromorphic system built with novel RRAM-based synapses. Each synapse consists of two RRAM devices of which one serves for long-term potentiation and the other for long-term depression. The RRAM devices are fabricated in-house with forming-free and continuous reset characteristics.Through a simulation tool based on the model of RRAM-based synapses, the proposed system has demonstrated the pattern recognition function with good performance.
\end{abstract}

Index Terms- Neuromorphic, Electronic Synapse, Resistive Random Access Memory (Rram), Long-Term Potentiation (Ltp), Long-Term Depression (Ltd)

\section{INTRODUCTION}

The dramatic development of silicon CMOS technology has enabled supercomputers capable ofrunning quadrillion $\left(10^{15}\right)$ floating-point operations per second, outperforming human brain in computing speed by a long shot. However, certain tasks that are simple to human, such as recognizing a particular person's face or voice, are still daunting to today's mainstream computing systems. A facial recognition computersystem would require a large number of micro-processors and a big database, and yet not often produce desirable results. Furthermore, power consumption has soared as a price of computation power gained and large computing systems today need sophisticated cooling systems for removal of generated heat. These facts have sparked lots of efforts in developing new forms of computing systems which can overcome these performance limits and also be more power efficient. One of the new computing forms well studied is the neuromorphic system, with encouraging results being demonstrated [1].

Neuromorphic systems are artificial computing systems based on principles obtained from biological nervous systems. In a nervous system, nerve cells, also known as neurons, are inter-connected and form neural circuits and networks [2]. The connections between neurons are called synapses, which have different weights and modulate inter-neuron signal strength accordingly. Neuromorphic systems realized to date in hardware have adopted various electronic devices for building synapses, such as static random access memory (SRAM) cells and floating-gate transistors [1]. Recently, resistive random access memory (RRAM) devices and their applications have been intensely studied [3] [4]. Electronic synapses built with RRAM devices have also been proposed [5] [6] [7].

However, due to the abrupt nature of the set operation in RRAM, electronic synapses built with RRAMdevices usually only show analog weight decrease, namely, long-term depression (LTD); whereas, analog weight increase, which is also called long-term potentiation (LTP), is difficult to realize. This paper proposes a neuromorphic system with RRAM-based electronic synapses that realize both LTP and LTD deterministically. Pattern recognition function is demonstrated by the proposed system.

The organization of this paper is as follows. Section II provides some background knowledge on neurons and synapses. Relevant work on electronic synapses is reviewed. The RRAM device used for constructing electronic synapses is also covered. Section III describes the novel electronic synapse constructed with two RRAM devices, one contributing positively (LTP) and the other contributing negatively (LTD).

The neuromorphic visual system architecture built with such electronic synapses is presented in Section IV. Good performance is demonstrated through simulation. A brief conclusion is given in Section V.

\section{BACKGROUND INFORMATION}

\section{A. Neuron and Synapse}

Almost all animals have nervous systems. The nervous system coordinates actions and transmits signals between different body parts, mainly through a special type of cells called neurons. A neuron generally consists of a soma, an axon, and dendrites (Figure 1).

The soma of a sending neuron creates and sends out action potentials, also called neural signals, which travel along the axon and arrive at synapses. Synapses (Figure 2a) modulates the signals according to their weights and transmit the modulated signals to the dendrites of the receiving neuron(s).

The sending neuron is called "presynaptic neuron" and receiving neuron "postsynaptic neuron". Changes of existing synapses, such as LTP and LTD which we study in this paper, and creations of new synapses are widely believed to underlie learning and memory, essential to human intelligence. Consequently, electronic 


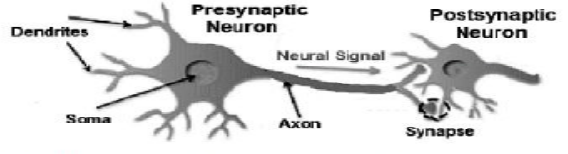

Figure 1. I/lustration of two connectedneurons in a nervous system.

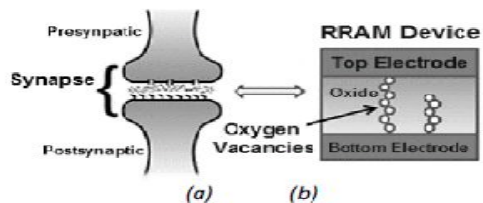

(b)

Figure 2. Analogy Between A Biological Synapse (A) And A RRAM Device (B).

synapses are very important components in a hardware neuromorphic system. Electronic synapses built with RRAM devices, compared with other approaches, have demonstrated many advantages including considerable area saving, low power consumption, high training speed, and non-volatility [1].

\section{B. RRAM Device}

The RRAMdevice can be modeled as a two-terminal electrically programmable resistor. As shown in Figure $2 b$, stacked in between the top electrode and bottom electrode is usually metal oxide. In general, the device operates on the soft electric breakdown of the metal oxide to create conductive filaments (trains of oxygen vacancies) that can be broken or reformed in subsequent programming (Figure 2b), thus exhibiting different electrical resistances.The variable resistancesare used to represent different states. The initial programming usually requires a relatively large voltage and the process is typically called "forming". The subsequent program process from low to high resistances is called "reset", and the programprocess from high to low resistances is "set". We have used a type of forming-free RRAM devices which do not needthe forming process and thus would require simplecircuit designs [4]. The device was made of a50-nm Pt bottom electrode, a 12-nm four-layer oxide stack of $\mathrm{HfO}_{\mathrm{x}} / \mathrm{TiO}_{\mathrm{x}} / \mathrm{HfO}_{\mathrm{x}} / \mathrm{TiO}_{\mathrm{x}}$ (from bottom to top), and a 50-nmTiN

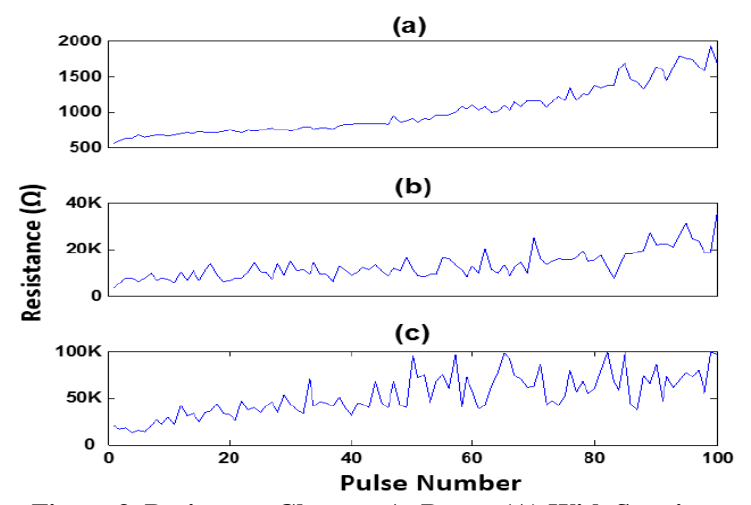

Figure 3. Resistance Changes At Reset: (A) With Starting Resistance $\sim 500 \Omega$ And Applied Voltage Pulses -1.1V/10ns, (B) With Starting Resistance $\sim 3 K \Omega$ And Applied Voltage Pulses $-1.2 \mathrm{~V} / 10 \mathrm{~ns}$, (C) With Starting Resistance $\sim 20 \mathrm{~K} \Omega$ And Applied Voltage Pulses -1.3V/10ns.

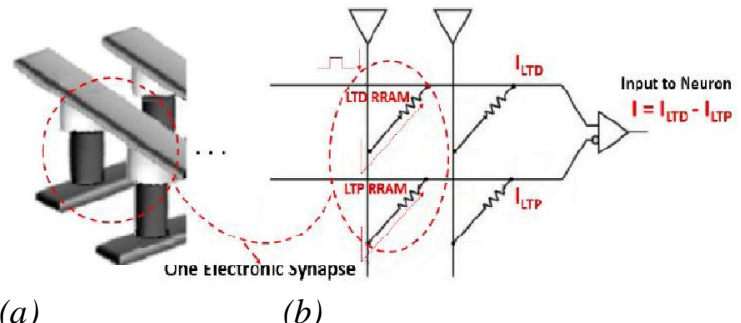

(a)

Figure 4. Schematics Of Proposed Synapse Built With Two RRAM Devices: (A)3D Crossbar Implementation, (B) Circuit Construction Under Read Operation.

top electrode. In electrical measurements, the voltage wasapplied to the top electrode (TiN) while the bottom electrode $(\mathrm{Pt})$ wasgrounded. The device has abrupt set, but it can be reset gradually. That means the device resistance can be increased gradually in a linear manner, as shown in Figure 3. This property is utilized for building electronic synapses, with the conductance (inverse of resistance) change emulating the gradual change of the synaptic weight.

\section{PROPOSED ELECTRONIC SYNAPSE}

Similar to their biological counterparts, electronic synapses change their weights in an analog fashion. The weights of electronic synapses are their conductances. RRAM devices that exhibit analog conductance change has been used to construct electronic synapses. However, RRAM devices usually only have gradual conductance decrease in the reset operation, so a synapse built with one RRAM device can show deterministic LTD behavior, but not LTP. That limitation poses certain constraint on the computing ability of constructed neuromorphic systems. To solve this problem, we propose an electronic synapse that exhibits deterministic LTP and LTD behaviors. The synapse consists of two RRAM devices, one for LTP and the other for LTD. Figure 4 shows the synapse in 3D crossbar implementation and circuit construction (at read/normal operation).During the training/writing process, conductances of both RRAM devices in a synapse can be decreased gradually through reset operations. The upper RRAM device is for LTD, because it contributes negatively to the output current as its conductance decreases. Reversely, the lower RRAM device contributes positively to the total current because of the minus operation, so it is for LTP.

The proposed electronic synapse have several advantages compared with the one based on a pair of phase change memory devices previously reported [8]. Because the RRAM device we adopt does not need forming, the extra circuit parts for forming operations are eliminated. Furthermore, the required set and reset pulse voltages are quite small and uniform, so the $1 \mathrm{R}$ crossbar array can be implemented. Energy consumption per spike can be reduced by orders of magnitude due to the low current and small time duration. The read or normal operation is illustrated in Figure 4b.For the RRAM write operation, the full 
write voltage is applied at the two lines connecting to the selected RRAM device; a half write voltage is applied at the other lines to avoid sneak paths and cross-talk effects.

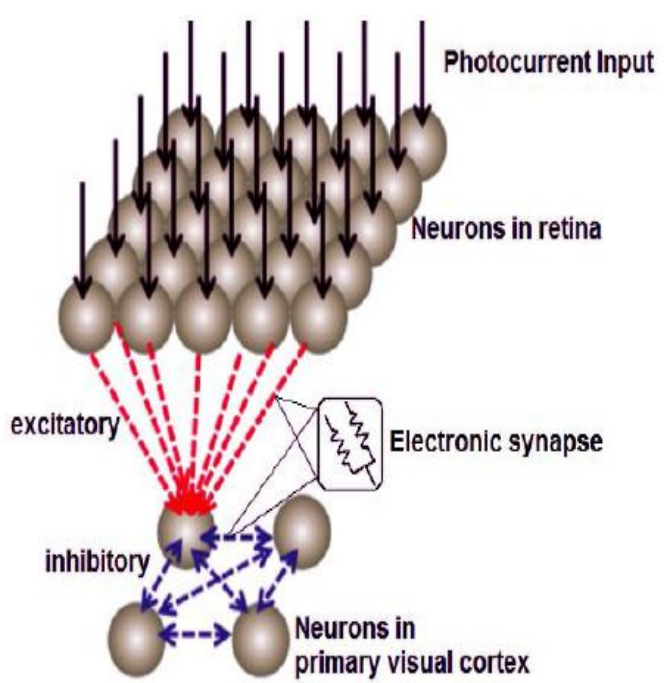

Figure 5. Illustration Of A Simple Neuromorphic Visual System With Two Layers Of Neurons: 25in The Retina (1 $1^{\text {st }}$ Layer $)$ And 4in The Primary Visual Cortex ( $2^{\text {nd }}$ Layer $)$.

\section{NEUROMORPHIC VISUAL SYSTEM}

\section{A. System Architecture}

We apply the electronic synapse to construct a simple neuromorphic visual system with 25 neurons in the retina ( $1^{\text {st }}$ layer) and 4 neuronsin the primary visual cortex ( $2^{\text {nd }}$ layer $)$ Total there are $4 \times 25+6=106$ RRAM-based synapses. It emulates the biological visual system with a winner-take-all architecture: under a certain input, if one neuron in the $2^{\text {nd }}$ layer fires first(becomes the winner), it then inhibits all the other neurons in the $2^{\text {nd }}$ layer (takes all). Additionally, the winning neuron feedbacks positively to the corresponding input neuron in the $1^{\text {st }}$ layer by increasing the weight (conductance) of their connection synapse.

As illustrated in Figure 5, the synapse is built with a pair of RRAM devices. The inhibitory synapses are colored in blue and excitatory synapses in red. The RRAM devices implemented would be grouped in crossbar arrays for high density. The neurons is based on the integrate-and-fire model and can be implementedin CMOS circuits or, for simple demonstration purpose, in software.

\section{B. Simulation Performance}

As the crossbar RRAM array is being fabricated and testing has been planned, we have developed a simulation tool for the neuromorphic system based on proposed RRAM synapse and neuron models. The simulation tool is constructed in $\mathrm{C \#}$ and run at the Windows Visual Studio platform. The RRAM device model has been presented previously [5]. A pattern of the Arabic number " 1 " is fed into the system for training. Figure 6 displays weights of the inter-layer synapses connecting to the four neurons in the $2^{\text {nd }}$ layers before and after training. We can see the pattern has been reflected in

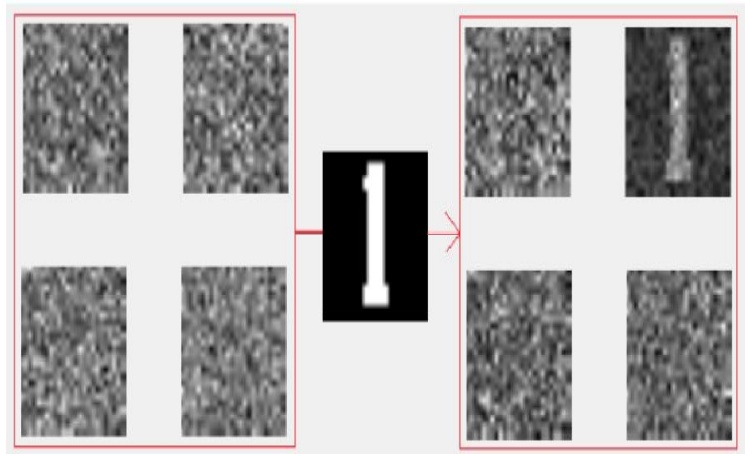

Figure 6. Relative Weight Diagrams of Excitatory Synapses Connecting To The Four Neurons In The 2 nd layer, In The Initial State (Left) And After 20 Training Cycles(Right) With The Input Pattern "1".

the weights of the synapses connecting to the right upper neuron after training. That means, if the future inputs to the system contain the trained pattern, this particular neuron will be activated and corresponding actions can be taken as a result of the recognition.

While we can conclude that the pattern recognition function has been demonstrated, there is still room for improvement. The devices show non-negligible variation during the recognition process, as reflected in the amount of noise displayed in the synapse weight diagram of the winning neuron after training (Figure 6). Methods for suppressing the effect of such variation are needed. A way of grouping a few RRAM devices in $3 \mathrm{D}$ to function as one device has been proved working [7], and it can be adopted here as well.

\section{CONCLUSION}

A neuromorphic system with novel electronic synapses based on RRAM devices has been proposed. Measurement data from the single devices are presented. The operation principles of the proposed system areexplained and also demonstrated through simulation.

\section{REFERENCES}

[1] D. Kuzum, S. Yu, H.-S. P. Wong, "Synaptic electronics: materials, devices, and applications," Nanotechnology, Vol. 24, pp. 382001, 2013.

[2] E. Kandel, J. Schwartz, T. Jessell, Principles of Neural Science, McGraw-Hill Medical: New York, 2000.

[3] H.-S. P. Wong, H.-Y. Lee, S. Yu, Y.-S. Chen, Y. Wu, P.-S. Chen, B. Lee, F.T. Chen, M.-J. Tsai, "Metal oxide RRAM,” Proc. IEEE, Vol. 100, pp. 1951-1970, 2012.

[4] Z. Fang, H. Y. Yu, X. Li, N. Singh, G. Q. Lo, D. L. Kwong, "HfOx/TiOx/HfOx/TiOx multilayer-based forming-free RRAM devices with excellent uniformity," IEEE Electron Device Letters, Vol. 32, No. 4, pp. 566 $568,2011$.

[5] S. Yu, B. Gao, Z. Fang, H. Yu, J. Kang, H.-S. P. Wong, "Low energy oxide-based electronic synaptic device for neuromorphic visual systems with tolerance to device variation," Adv. Mater., Vol. 25, pp. 1774 - 1779, 2013. 
[6] S. Yu, B. Gao, Z. Fang, H. Yu, J. Kang, H.-S. P. Wong, "Stochastic learning in oxide binary synaptic device forneuromorphic computing," Vol. 7, pp. 186, Front. Neurosci. 2013.

[7] B. Gao, Y. Bi, H.-Y. Chen, R. Liu, P. Huang, B. Chen, L. Liu, X. Liu, S. Yu, H.-S. P. Wong, and J. Kang, "Ultra-low-energy three-dimensional oxide-based electronic synapses for implementation of robust high-accuracy neuromorphic computation systems," ACS Nano, VOL. 8, No. 7, pp. 6998 - 7004, 2014.

[8] M. Suri, O. Bichler, D. Querlioz, O. Cueto, L. Perniola,V. Sousa, D. Vuillaume, C. Gamrat, and B. DeSalvo, "Phase change memory as synapse for ultra-denseneuromorphic systems: application to complex visualpattern extraction," IEEE Int. Electron DevicesMeeting (IEDM), pp 4.4.1-4, 2011. 